\title{
Validation of a Coupled Thermal-Electromagnetic Quench Model for Accelerator Magnets
}

\author{
Nikolai Schwerg, Bernhard Auchmann, and Stephan Russenschuck
}

\begin{abstract}
Quench simulation in superconducting magnets is a challenging task due to the interdependence of thermal, electrical, and magnetic phenomena. We present a new quench-simulation module in the CERN magnet-design program ROXIE. Thermal, electrical, and magnetic models are solved simultaneously. The integrated model helps to single out the impact of different phenomena. We can thus reach a deeper understanding of measured quench behavior. Moreover, the magnet-design process is improved due to the implementation within an integrated design and optimization environment. We compare simulations and measurements of the LHC main dipole magnet.
\end{abstract}

Index Terms-Quench simulation.

\section{INTRODUCTION}

$\mathbf{Q}$ UENCH protection is an important issue in the design of future high-field or fast-ramping superconducting magnets. Protection strategies must be addressed in the earliest stages of development, in order to control peak-temperature and internal voltages. We will show that it is important to treat all involved phenomena (thermal, electrical, and magnetic) in a coupled way. The integrated model allows to single out the impact of different phenomena, e.g., quench-back, quenchpropagation, heaters, and iron saturation. We can thus estimate the impact of each individual effect and come to a deeper understanding of a measured quench behavior. The efficiency of quench-protection strategies can be assessed by means of simulations.

Given the uncertainties in many material parameters, it must be noted that quench simulation is an ill posed problem. There are by far more model parameters than validation criteria from measurement (current decay, voltages). Section III gives an example how different models can yield equivalent current decay curves. We conclude that quench models must not be abused as a fit to measurement. Great care needs to be taken in the determination of realistic material parameters. All involved phenomena have to be modeled diligently, and their mutual coupling must be adequately represented in the numerical algorithm.

Over the past decades, quench-simulation routines used to decouple the electromagnetic field problem from the thermal simulation. As examples we mention semi-analytical approaches in [1]-[3], and a model that employs a commercial network solver

Manuscript received August 25, 2007

The authors are with CERN, Beijing 100101, China (e-mail: nikolai. schwerg@cern.ch).

Color versions of one or more of the figures in this paper are available online at http://ieeexplore.ieee.org.

Digital Object Identifier 10.1109/TASC.2008.920682

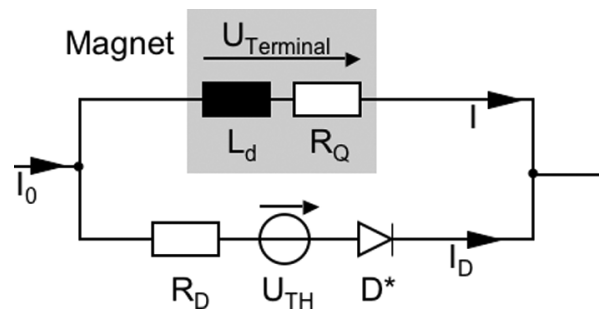

Fig. 1. Simplified electrical network of the magnet and the external circuit.

for the thermal and electrical models [4]. A FEM model for the calculation of quench propagation was used in [5], [6]. The limitation of these methods is reached when strong saturation effects in the yoke occur, which cannot be represented by equivalent inductances and scaled field-maps.

A strong coupling of field calculation, thermal simulation, and electric-circuit analysis is presented in [7] for solenoidal magnets. The thermal problem is solved on the same finite-element mesh as the electromagnetic problem. The required meshing of the coil, air domain, and iron yoke makes this approach computationally expensive for the use in an integrated design process for accelerator magnets.

Our quench model is briefly introduced in Section II. The main focus of this paper is on the discussion of simulation results and the comparison to measurement in Section III.

\section{Quench Calculation Routine}

We briefly introduce our quench model. More detailed information on the algorithms can be found in [8].

We assume that the transition between the superconducting and resistive states in a conductor is abrupt (in the present state of implementation we neglect current-sharing phenomena). The transition occurs when the local magnetic induction, the temperature, or the current density exceed critical values. This fact reflects the need for a coupled magnetic, thermal and electric simulation.

The magnetic field computation is carried out in 2-D, using the coupling method of boundary- and finite elements (BEM-FEM coupling method) to take into account the non-linear iron magnetization [9]. The differential inductance $L_{\mathrm{d}}$ of the magnet is determined from the linked flux $\Psi_{1}$ in the coils, $L_{\mathrm{d}}=\partial \Psi_{1} / \partial I$. Induced voltages are calculated from $U=-\partial \Psi_{1} / \partial t$. Moreover, the magnetic model computes eddy-current losses $P_{\text {Loss }}$ in the Rutherford-type cable, see [10], which act as heat sources in the thermal model.

The excitation current is calculated from a simplified electrical network as shown in Fig. 1. The network consists of the 
resistance $R_{\mathrm{Q}}$ due to the normal zone, the differential inductance $L_{\mathrm{d}}$, and a cold by-pass diode. The diode is modeled by the forward threshold voltage $U_{\mathrm{TH}}$ and the forward resistivity $R_{\mathrm{D}}$. The circuit is powered by a constant current $I_{0}$.

The resistivity of a quenched conductor depends on the magnetic induction due to magneto-resistivity, and on the temperature, where the residual resistivity ratio (RRR) is used to model the temperature dependence. The power $P_{\mathrm{Ohm}}$, that is dissipated in the conductor's resistivity, acts as a heat source in the thermal model.

The potential to ground of each conductor is calculated as the sum of ohmic and induced voltages, taking into account the exact winding scheme.

The simulation of temperature and quench-propagation is based on a 3-D model of the coil. Field variations in the coil ends are neglected, the ends of the straight section are assumed to be connected seamlessly. The coil is subdivided longitudinally into $N$ elements. The magnetic-field distribution and the current density are assumed constant in longitudinal direction. They are obtained from the 2-D BEM-FEM calculation.

Let $i$ be the index of conductors in the cross-section and $j$ the index of longitudinal subdivisions. The temperature in an element is computed from the discrete formulation of the heat balance equation:

$$
\begin{aligned}
\frac{\partial T_{i, j}}{\partial t}=\frac{1}{\rho_{i, j} C\left(T_{i, j}\right)}( & P_{i, j}-K_{\mathrm{t}} l_{z}\left(T_{i+1, j}-2 T_{i, j}+T_{i-1, j}\right) \\
& \left.-\frac{K_{1}}{l_{z}}\left(T_{i, j+1}-2 T_{i, j}+T_{i, j-1}\right)\right)
\end{aligned}
$$

Here $C\left(T_{i, j}\right)$ denotes the averaged non-linear heat capacity, $\rho_{i, j}$ the mass density, and $l_{z}$ the length of the element. The heating power $P_{i, j}$ consists of induced losses $P_{\text {Loss }}$, ohmic heating $P_{\mathrm{Ohm}}$, and quench-heater power. Specific heater delays and discharge curves of heaters are considered.

In the current state of implementation, heat transfer occurs across the broad side of neighboring cables and along the cable. The longitudinal and transversal heat-transfer coefficients $K_{1}$ and $K_{\mathrm{t}}$ are prescribed such that the expected turn-to-turn quench-propagation delay and the longitudinal quench propagation velocity are reproduced.

The model does not yet take into account cooling of the superconducting cable. By means of user-supplied empirical parameters, e.g., heat transfer coefficients and heater settings, some cooling effects are nonetheless represented.

The update of the magnetic field is computationally more expensive than a time-step in the thermal model. Time-constants in the thermal model, however, are much shorter than those of the eddy currents in the magnetic model. It is therefore reasonable to foresee a weak coupling between thermal and magnetic computations, updating magnetic values only when the excitation current has changed significantly [8].

\section{RESULTS}

The quench routine is demonstrated and validated at the example of the LHC main bending magnet [11]. We review relevant properties of the magnet, and specify the used parameters.
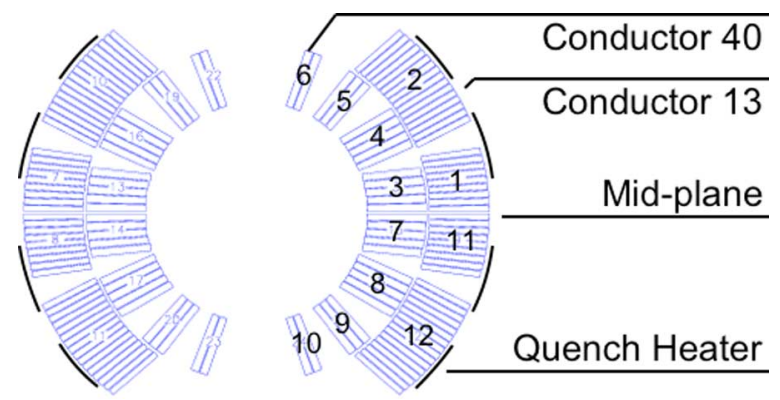

Fig. 2. Coil blocks and quench heater positions in one aperture of the LHC main bending magnet.

The dipole features two apertures in a common iron yoke. The coils in each aperture are subdivided in an upper and a lower pole. Each pole consists of 6 conductor-blocks per quadrant, arranged in two-layers. Fig. 2 shows one aperture. Saturation has a relatively small impact on the differential induction, which varies only by $51 \%$ over excitation. For thermal calculations an average conductor length between the two coil ends of $14.57 \mathrm{~m}$ is used.

The coil is wound from Rutherford-type Nb-Ti cable. The critical current density $J_{c}(B, T)$ in the strands is given by a fit to measurement [12]. The magnet operates at $1.9 \mathrm{~K}$. The residual resistivity ratio (RRR) of the copper matrix in the strands is in the range of 150 to 250 [13]. The inter-strand contact resistances of the inner and outer layer cables are about $30 \mu \Omega$ and $60 \mu \Omega$, respectively [14]. At a ramp rate of $7.5 \mathrm{mT} \cdot \mathrm{s}^{-1}$, AC losses of $180 \mathrm{~mW}$ per meter can be expected in the magnet [11]. During a quench, theses losses increase by many orders of magnitude, and cause quench-back [15].

The longitudinal and transversal heat transfer coefficients are set to $0.2 \mathrm{~W} \cdot \mathrm{m} \cdot \mathrm{K}^{-1}$ and $0.3 \mathrm{~W} \cdot \mathrm{m}^{-1} \mathrm{~K}^{-1}$, respectively. In absence of induced losses, this corresponds to longitudinal quench propagation velocities of $10-20 \mathrm{~m} \cdot \mathrm{s}^{-1}$ and a turn-toturn delay of $20 \mathrm{~ms}$ at nominal current (11.85 kA) [5].

The quench protection consists of a detection system, a cold by-pass diode and quench heaters placed on the outer layer of the coil as indicated in Fig. 2. The threshold voltage of the detection system is $0.1 \mathrm{~V}$ [16]. The forward voltage of the diode is assumed to be constant at $8 \mathrm{~V}$. Quench heaters are fired after a delay of $10 \mathrm{~ms}$ for signal validation [16]. The timing of the different heaters may vary by up to $10 \mathrm{~ms}$ [5]. A capacitor is discharged over the resistance of the heater strip, resulting in an exponential voltage decay [15]. The time constant for the dissipated power is about $37.5 \mathrm{~ms}$ [5]. Measurements indicate that a heater-provoked quench at $1.5 \mathrm{kA}$ occurs around $80 \mathrm{~ms}$ after the heaters are fired. At nominal current the delay reduces to 35 ms [17]. Since the quench heaters are modeled as heat sources inside the conductors, a delay-time of $30 \mathrm{~ms}$ is foreseen that represents the heat-transfer time from the heater to the coil. Together with a power-amplitude of $20 \mathrm{~W} \cdot \mathrm{m}^{-1}$ per conductor, the measured heater-delays (at $1.5 \mathrm{kA}$ and nominal currents) are reproduced.

\section{A. Quench at Nominal Current}

We consider a quench at the nominal current of $11.85 \mathrm{kA}$. The quench origin is located in the outer layer in between two heater strips (conductor 13). It is centered longitudinally. 


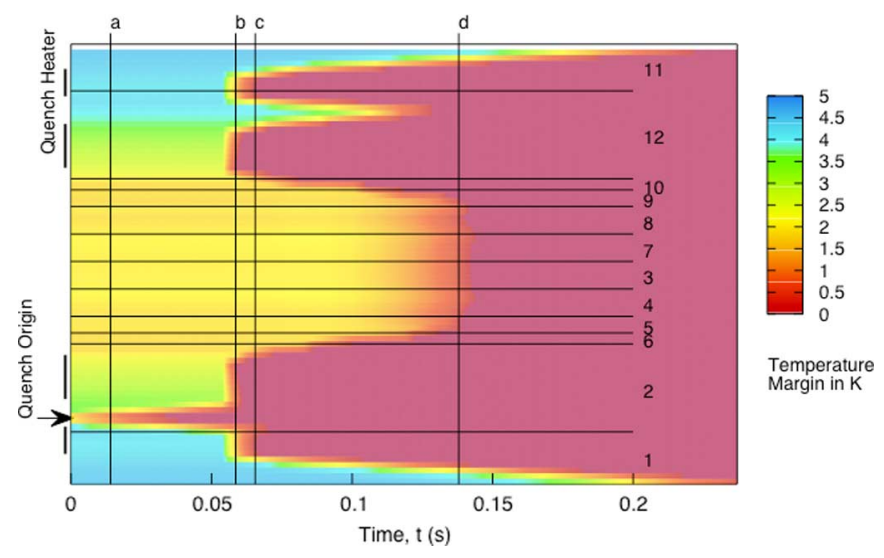

Fig. 3. Temperature margin to quench in the conductors versus time. The numbers correspond to the block numbers in Fig. 2. From the evolution of the temperature margin we can distinguish quench propagation, quench heater delay, and quench-back
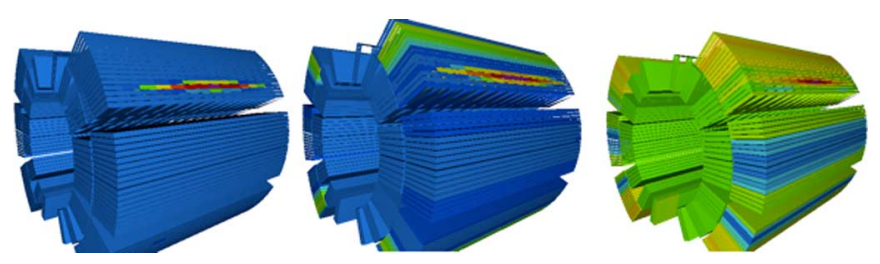

Fig. 4. Illustrated temperature distribution over one aperture of the magnet. We distinguish quench propagation, quench-heater firing, and quench-back. The coil is scaled 1:100 in axial direction.

Fig. 3 shows the temperature margin to quench in each conductor at the magnet's centre as a function of time. Three different phenomena can be distinguished: Quench propagation, quench-heater delay, and quench-back. After the quench has been initiated, it propagates transversally and longitudinally. The detection voltage is reached at $14.1 \mathrm{~ms}$ (marked with 'a' in Fig. 3) and $10 \mathrm{~ms}$ later the quench heaters are fired. After the pre-defined $30 \mathrm{~ms}$, the dissipated heat starts to decrease the quench margin in the conductors covered by heaters. Conductors covered by the high-field heaters quench at $58.6 \mathrm{~ms}$ (b). Conductors covered by low-field heaters reach the critical temperature $7 \mathrm{~ms}$ later (c). With the first conductors quenched, the diode threshold voltage is reached and the current in the magnet starts to decrease. Eddy-current losses create additional heating. The inner layer is quenched by magnetic quench-back at around $140 \mathrm{~ms}(\mathrm{~d})$. The quench propagation in the outer layer is also accelerated by the induced losses. After about $260 \mathrm{~ms}$ the magnet is fully quenched.

Fig. 4 shows the coil temperature at three different stages of the quench. On the left, the initial quench zone propagates transversally and longitudinally due to thermal conduction. In the middle, the quench heaters have been fired and the conductors covered by the high-field heaters are resistive. The conductors under the low-field heaters are not yet quenched. The figure on the right shows the magnet after quench-back. The coil is almost completely resistive.

We note that the term "quench-propagation velocity" is ambiguous. In the absence of quench heaters and neglecting induced losses, the velocity depends amongst others on the thermal conductivity and on the excitation of the magnet. With

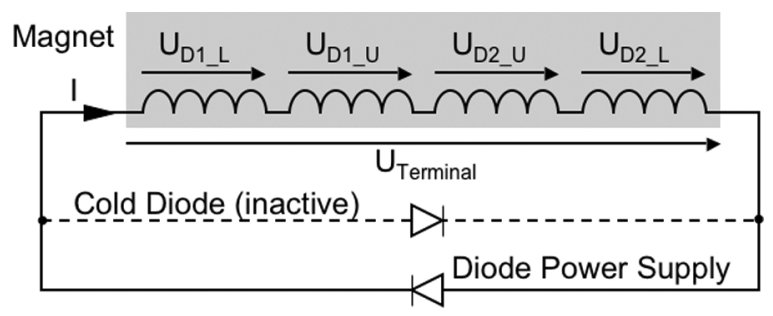

Fig. 5. Electrical circuit of the magnet measurement test station after the current source is switched off. D1_L denotes the lower pole in aperture 1. D2_U denotes the upper pole in aperture 2 .

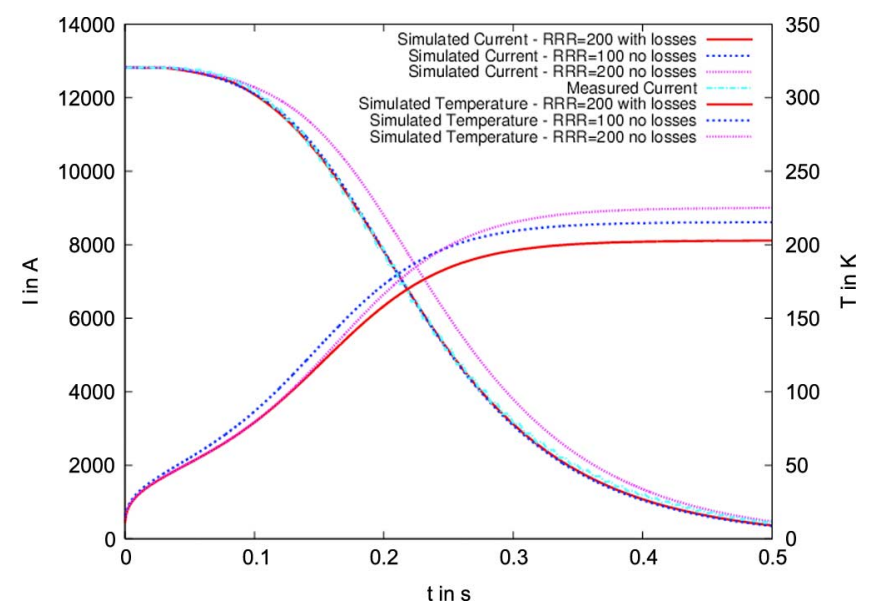

Fig. 6. Measured and simulated current decrease for varying simulation parameters. Simulated peak temperature.

our model assumptions, and with all conductors infinitesimally close to the critical surface, the quench-propagation velocity will be infinite. Furthermore, considering induced losses, the velocity depends on the ramp rate. In case of a magnetic quench-back and in the presence of quench heaters, the quench does not propagate continuously.

\section{B. Validation With Measurements}

We compare our simulations to the measured current and voltage signals during a training quench of magnet MB2381 [18]. For this measurement, an external free-wheeling diode is used, which is mounted with opposite orientation as compared to the magnet's cold diode. The current source is switched off as soon as a quench is detected. Thus the current commutes into the warm diode. The terminal voltage $U_{\text {Terminal }}$ of the magnet changes sign between the positive voltage over $R_{\mathrm{Q}}$ before quench detection and the negative forward voltage of the diode after detection. The negative terminal voltage leads to a faster current decay than under operational conditions in the LHC string of magnets. From the measured terminal voltage we derive the diode properties, i.e., a diode threshold voltage of $0.7 \mathrm{~V}$ and a forward resistance of about $390 \mu \Omega$. The electrical circuit used for the simulation is shown in Fig. 5. The quench starts at a current level of $12.82 \mathrm{kA}$ in conductor 40 of pole D1_U.

Fig. 6 shows the simulated current decays for different RRR values, with and without induced losses in the conductors. The results are compared to the measured current decay. Simulation 


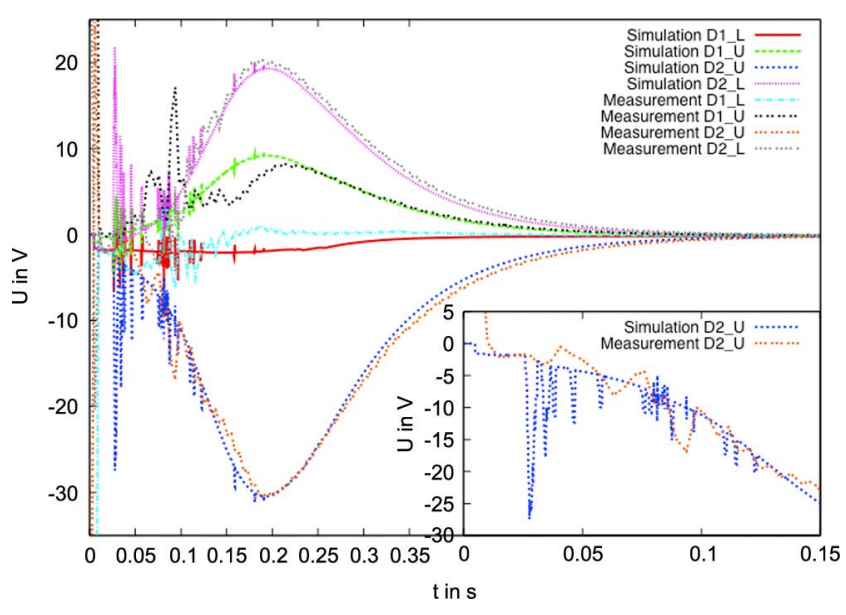

Fig. 7. Measured and simulated pole voltages.

1 uses a RRR of 100 and neglects induced losses. Simulation 2 uses a RRR of 200 while taking induced losses into account. Both simulations show a good agreement with measurement. The measurement data cannot be reproduced for a RRR value of 200 if losses are neglected. Although the simulations 1 and 2 yield a similar current decay, the peak temperatures differ by $10 \mathrm{~K}$. We conclude that a good reproduction of the current decay does not imply that the quench behavior is modeled accurately.

The measured and simulated voltages across the four poles of the two-in-one magnet are shown in Fig. 7. Small differences in the timing of the quench heaters over the four poles cause asymmetric voltage distributions. To reproduce the asymmetry, the quench heaters in our model were de-tuned by less than $2 \mathrm{~ms}$.

The measured voltage, as well as the simulation, show ripples during the first $100 \mathrm{~ms}$ (see inset in Fig. 7). We explain this by the fact that every conductor turning resistive causes a sudden increase in ohmic voltage. The magnet's terminal voltage, however, is clamped to the forward voltage of the diode. Neglecting internal capacities, the increase in ohmic voltage must be distributed evenly over the four pole inductances, resulting in a sudden change of all voltages. Once all conductors are quenched, the ohmic voltage changes smoothly with temperature and the measured and simulated curves are smooth. Note that the sampling rate of the measurement is $227 \mathrm{~Hz}$ whereas the adaptive step-size control of the simulation yields sampling rates of about $10 \mathrm{kHz}$. Taking into account current-sharing would smoothen the ripples in the simulation.

\section{CONCLUSION}

We have presented a quench model that is based on 2-D electromagnetic field computation, a thermal 3-D finite-difference model, and an electrical network. The effects of iron-saturation and induced losses are taken into account. The model has been validated with measurements of the LHC main dipole. By validation we understand the accurate reproduction of measurement data, under the condition that all relevant phenomena are adequately modeled. The determination of realistic model parameters is the most difficult task in a simulation. We expect that our approach leads to improved estimates of peak temperatures and internal voltages.

The integration of the model into a magnet-design environment allows to assess the efficiency of quench-protection strategies by means of simulation. Moreover, we can use the model to reach a deeper understanding of measured quench behavior. The method will be extended to deal with non-linear, and temperature dependent heat-transfer coefficients. Cooling by liquid helium will equally be implemented.

\section{REFERENCES}

[1] M. N. Wilson, Computer Simulation of the Quenching of a Superconducting Magnet Rutherford High Energy Laboratory, RHEL/M 151, 1968, Internal Report.

[2] M. N. Wilson, "Superconducting Magnets," in Monographs on Cryogenics. New York, USA: Oxford University Press, 1983.

[3] M. Canali and L. Rossi, Dynque : A Computer Code for Quench Simulation in Adiabatic Multicoil Superconducting Solenoids. Milano, Italy, : , June 1993, INFN-TC- 93-06, INFN.

[4] D. Hagedorn and F. Rodriguez-Mateos, "Modelling of the quenching process in complex superconducting magnet systems," IEEE Transactions On Magnetics, vol. 28, no. 1, pp. 366-369, Jan. 1992, CERN. Geneva, Switzerland: IEEE.

[5] F. Sonnemann, "Resistive transition and Protection of LHC Superconducting Cables and Magnets," Ph.D. dissertation, RheinischWestfalische Technische Hochschule Aachen, Aachen, Germany, May 2001.

[6] S. Caspi et al., "Calculating quench propagation with ANSYS," IEEE Trans. Appl. Supercond., vol. 13, no. 2, pp. 1714-1717, June 2003, IEEE.

[7] G. J. C. Aird, J. Simkin, S. C. Taylor, C. W. Trowbridge, and E. Xu, "Coupled transient thermal and electromagnetic finite element simulation of quench in superconducting magnets," in Proceedings of the ICAP 06, Charmonix, France, October 2006, pp. 69-72, submitted for publication.

[8] N. Schwerg, B. Auchmann, and S. Russenschuck, "Quench simulation in an integrated design environment for superconducting magnets," IEEE Trans. Magn., June 2007, sent in for publication.

[9] S. Russenschuck, Electromagnetic Design and Mathematical Optimization Methods in Magnet Technology, 4th ed. Geneva, Switzerland: CERN, Sept. 2006, ISBN: 92-9083-242-8.

[10] S. Russenschuck, B. Auchmann, and R. de Maria, in Calculation of Timetransient Effects With the CERN Field Computation Program ROXIE, August 2007, this conference.

[11] O. Brüning et al., LHC Design Report-The LHC Main Ring. Geneva, Switzerland: CERN, 2004, vol. 1, Eds..

[12] L. Bottura, "A practical fit for the critical surface of NbTi," IEEE Trans. Appl. Supercond., vol. 10, no. 1, pp. 1054-1057, March 2000.

[13] Z. Charifoulline, "Residual resistivity ratio (RRR) measurements of LHC superconducting NbTi cable strands," IEEE Trans. Appl. Supercond., vol. 16, no. 2, pp. 1188-1191, 2006.

[14] D. Leroy, "Review of the R\&D and supply of the LHC superconducting cables," IEEE Trans. Appl. Supercond., vol. 16, no. 2, pp. 1152-1159, 2006.

[15] F. Rodríguez-Mateos and F. Sonnemann, "Quench heater studies for the LHC magnets," in LHC Project Report 485. Geneva, Switzerland: CERN, August 2001 [Online]. Available: http://cdsweb.cern.ch/ record/514347

[16] R. Denz, "Electronic systems for the protection of superconducting elements in the LHC," IEEE Trans. Appl. Supercond., vol. 16, no. 2, pp. 1725-1728, 2006.

[17] P. Pugnat, Measurement of Quench Heater Delays. July 2007, Private Communication.

[18] V. Chohan and E. Veyrunes, Measurement Data From the Training Quench of Magnet MB2381 at 12.82 kA. July 2007, Private Communication. 